\title{
La gestión de recursos humanos, el capitalismo y el socialismo
}

\section{Human resource management, capitalism and socialism}

Ing. Lubiza Osio Havriluk

Universidad de Carabobo, Venezuela

Autor para correspondencia: Lubiza@gmail.com

Fecha de recepción: 10 de Noviembre de 2016 - Fecha de aceptación: 10 de Enero de 2017

\section{Resumen}

El tema de la Gestión de Recursos Humanos (GRRHH), observado desde dos modelos divergentes como lo son el Capitalismo y el Socialismo, permite debatir sobre las diferencias fundamentales de este quehacer en cada modelo. En tal sentido, el objetivo de este ensayo viene dado por comprender como opera la Gestión de Recursos Humanos en estos modelos. Para que esto fuese posible la metódica requerida precisó de un nivel descriptivo de apoyo fundamentalmente documental, que permitiera explanar las nociones básicas sobre el tema, revisar el tema del Trabajo y la GRRHH, para luego explorar el Pensamiento Social de las Relaciones Laborales, observando el caso de Venezuela en el Socialismo del Siglo XXI; para finalmente dar luces sobre cuál es el papel de la gerencia de recursos humanos en cada modelo.

Palabras clave: Gestión de Recursos Humanos; Capitalismo; Socialismo; Socialismo del Siglo XXI

\begin{abstract}
The issue of Human Resources Management (HRM), observed from two divergent models such as Capitalism and Socialism, allows discuss the fundamental differences of this task in each model. In this sense, the purpose of this test is given to understand how it operates the Human Resources Management in these models. To make this possible the methodical required said in a fundamentally documentary descriptive level of support, which would allow grading the basic notions on the subject, revisit the issue of Labour and HRM, then explore the Social Thought of Labor Relations, observing the if Venezuela in the XXI Century Socialism; to finally shed light on what is the role of human resource management in each model.
\end{abstract}

Key words: Human Resource Management; Capitalism, Socialism; XXI Century Socialism 


\section{Introducción}

Empecemos por definir que es el capitalismo. Para Weber (1978)

Existe el capitalismo dondequiera que se realiza la satisfacción de necesidades de un grupo humano, con carácter lucrativo y por medio de empresas, cualquiera que sea la necesidad de que se trate; especialmente diremos que una explotación racionalmente capitalista es una explotación con contabilidad de capital, es decir, una empresa lucrativa que controla su rentabilidad en el orden administrativo por medio de la contabilidad moderna, estableciendo un balance (en línea).

Para Marx y Engels, "el capitalismo basaba su existencia en la pervivencia de divisiones de clase que eran beneficiosas para algunos sectores (los sectores burgueses) y perjudiciales para otros (los sectores proletariados, trabajadores y explotados por los primeros)" (Definición ABC, 2015a: en línea). Se puede definir al capitalismo como un sistema económico, donde el capital prevalece sobre el trabajo y es la base de la riqueza. Dentro de este régimen la propiedad privada y el libre comercio son las dos bases elementales. Para que este exista, deben existir tres actores fundamentales dentro del mercado: los capitalistas, los trabajadores y los consumidores.

Para Marx (2014), el capitalismo representaba la forma de explotación del hombre por el hombre, donde el trabajo alienaba al ser humano y lo llevaba a ser un objeto más de la fábrica. Desde su visión, el capitalismo degeneraba la economía y la sociedad y la llevaba a un caos perverso que la aniquilaría. Su propuesta era un Comunismo, como forma de organización que permitiera a todos los trabajadores tener acceso a las riquezas. Esto planteaba la formación de una sociedad sin clases sociales, donde los medios de producción son de propiedad común y el poder lo tendría la clase trabajadora.

Por otro lado, existe el socialismo, creado por Marx como una vía para la construcción del comunismo. Se define el socialismo como "un sistema de organización social y económica que se basa en la propiedad y administración colectiva o estatal de los medios de producción y se propone que progresivamente las clases sociales desaparezcan" (Definición ABC, 2015b: en línea). En el socialismo se reemplaza la propiedad privada de los medios de producción por la propiedad colectiva de estos. Además, se instaura la dictadura del proletariado para realizar esta tarea y lanza las bases para una sociedad superior basada en la abundancia, la igualdad social y el pleno desarrollo del individuo. Para Marx (2014), el socialismo no elimina las contradicciones de clase sino que crea las condiciones para eliminarlas.

Estas tres visiones, teniendo el capitalismo y el comunismo como posiciones completamente contrapuestas, plantean dos tipos de economías, y sociedades donde las visiones empresariales difieren grandemente y que generarían empresas realmente diferentes, con comportamientos distintos y manejo del Recurso Humano desde perspectivas distintas. Entonces, como lo indica Delgado (2004: 9) "la acción práctica de facilitar y garantizar el uso adecuado de los recursos humanos al interior de la organización es una consecuencia obvia de la forma como se asume la producción y la dinámica de las relaciones industriales”. 
En el caso del Capitalismo, según Delgado (2004:9), se debe "asociar la problemática de la gestión de recursos humanos al permanente estado de valor supremo organizacional: ser productivos". Desde esta perspectiva, la gestión de Recursos Humanos está destinada a promover todo un ambiente lo suficientemente sólido que permita el desarrollo de la productividad y la calidad. No parece el mismo caso bajo la premisa del comunismo.

En el caso del Socialismo, Vilches (2011) indica:

Países democráticos cuyos gobiernos son socialistas moderados que han sido exitosos, Brasil, Suecia son solo un ejemplo, abrieron el mercado y comprendieron hace mucho tiempo que el Estado debe ser más que benefactor, deber ser posibilitador, contrario a esto existen gobiernos socialistas más radicales en países como Venezuela que entre otras cosas se caracteriza por ser autoritario, centraliza el poder y quisiera eliminar del todo a la empresa privada. (En línea)

Esto quiere decir que dependiendo de cómo se implante este último modelo, se tendrán mejores o peores resultados. Este autor, indica que el fracaso en los países socialistas se debe a "el gasto público desmedido de los gobiernos, beneficios a los empleados públicos, una burocracia gigante e ineficiente, poca productividad" (Vilchez, 2011: en línea). Cuando apunta al Socialismo en Venezuela expone que:

En nuestra región podemos mencionar un experimento de socialismo más radical, el Socialismo del siglo XXI que impulsa Chávez en Venezuela, y que como sabemos, aún con 12 años continuos en el poder y con la entrada de miles de millones producto de los petrodólares, no ha sido capaz de levantar los índices de equidad, seguridad y desarrollo, una nación que contaba con 40 años de democracia en evolución, pasó a tener un gobierno socialista radical, autoritario, ineficiente, que coarta las libertades, aumenta la burocracia por puro clientelismo, hace expropiaciones por doquier, amenaza a la empresa privada, estatiza todo lo que puede y tiene a más de la mitad de la población en su contra (Vilchez, 2011: en línea).

Aunque hay muchos ejemplos fallidos, como Grecia, Portugal, Venezuela, hay gobiernos socialistas exitosos, modernos y moderados, como los de los países escandinavos. Estos se caracterizan por ser honestos, entienden la importancia de la empresa privada, que es necesaria la libertad económica y política. En el caso del socialismo podríamos entender que se puede conseguir progreso y que pueden darse modelos empresariales mucho más abiertos y equitativos, que promueva las prácticas de la GRRHH actuales e innovadoras, que impulsen a la productividad y la calidad. En este sentido, para Delgado (2004: 9), la GRRHH es "un acto singular o marginal, esencial o accesorio en atención al rol que ésta representa como generador de las acciones con capacidad de agregarle valor al proceso que genera la acción competitiva".

\section{El Trabajo y la GRRHH}

En la sociedad postindustrial el trabajo ha perdido la centralidad. Elementos como: "una tendencia progresiva de descentralización hacia la empresa, con procesos de flexibilización, un marcado valor a la calificación profesional" (Delgado, 2004:17), la inclusión de las Tecnologías de Información y Comunicación a las organizaciones, han contribuido a que las formas de 
trabajo, así como su organización cambien. Es importante indicar que "los cambios en la naturaleza del trabajo, no son exclusivos de las economías prósperas... en América Latina se está dando un rápido proceso de cambio, impulsado por la demanda de mercados de exportación y los procesos de liberación central" (Delgado, 2014: 18). Delgado también indica (2014) que en ese proceso de transformación, el trabajo recobra vitalidad en tanto se revaloriza por la acción de las competencias implícitas en el proceso de trabajo, cuya complejidad, supone acciones que van mucho más allá de la simple utilización de las habilidades y destrezas, para encontrar en la adaptabilidad los comportamientos y la comunicación intra y extra empresa un conector de valoración de sus productos. (p. 20-21)

Otra definición importante es, qué es la Gestión de los Recursos Humanos:

Es el instrumento de acción administrativa a través de la cual, la organización del trabajo formaliza, viabiliza e instrumenta las acciones de provisión, aplicación, mantenimiento, desarrollo y control de los recursos humanos, dentro de una concepción que apuntala los requisitos y demandas implícitas en la organización del trabajo (Delgado, 2014:9)

Las áreas de estudio de la Gestión de Recursos Humanos, según Werther y Davis (1991) son:
a) Preparación y selección;
b) Desarrollo y evaluación;
c) Compensaciones y protección;
d) Relaciones con los empleados.

Ellas abarcan todo lo relacionado a las actividades que permiten que el personal sea productivo en una organización pero que también sea considerado un elemento importante dentro de esta. Claro está, que esto dependerá de las políticas de RRHH que tenga la organización.

Ante todo lo anterior, podemos hacernos la siguiente pregunta ¿hacia dónde va el trabajo y la gestión de los recursos humanos? "Es difícil contestar, pero no hay duda que ella será la consecuencia de las demandas de la sociedad, que ha sido ineludiblemente atropellada por toda una cultura más mediática, cambios tecnológicos, y tendencias globalizantes" (Delgado, 2014:17).

\section{Pensamiento Social de las Relaciones Laborales}

A fin de dar respuesta a la siguiente interrogante: ¿Son pertinentes los modelos contemporáneos de gestión de Recursos Humanos en sistemas no capitalistas? se procede a analizar el pensamiento social de las Relaciones Laborales, en los Modelos:

a) Cibernético Abierto, por encontrarnos en la Sociedad de la Información en el momento actual en los países capitalistas y el los socialistas modernos, y

b) el Modelo de Cambio Social Histórico Dialectico con enfoque Marxista, existente en países comunistas y algunos socialistas. 
Con el objetivo de estudiar dos modelos contrapuestos. Todo el material teórico se toma de la Tesis Doctoral de Delgado (2004:73-78):

Tabla 1. La Gestión de Recursos Humanos y los modelos contrapuestos

\begin{tabular}{|c|c|c|}
\hline Aspectos & Modelo Cibernético Abierto & $\begin{array}{c}\text { Modelo de Cambio Social Histórico } \\
\text { Dialectico }\end{array}$ \\
\hline $\begin{array}{l}\text { Definición } \\
\text { Clave }\end{array}$ & $\begin{array}{l}\text { Es el resultado de un complejo de actividades } \\
\text { públicas y privadas que operan en un medio } \\
\text { ambiente dominado por la preocupación de la } \\
\text { distribución de recompensa a los empleados, por } \\
\text { los servicios prestados y por las condiciones de } \\
\text { trabajo en que prestan tales servicios. }\end{array}$ & $\begin{array}{l}\text { Las Relaciones Industriales estudian los } \\
\text { procesos de control sobre las relaciones } \\
\text { laborales. }\end{array}$ \\
\hline $\begin{array}{l}\text { Variable } \\
\text { Focal }\end{array}$ & $\begin{array}{l}\text { Distribución de recompensas económicas, } \\
\text { psicológicas y sociales. }\end{array}$ & El Control. La Dialéctica capital-trabajo \\
\hline $\begin{array}{l}\text { Actores } \\
\text { sociales }\end{array}$ & $\begin{array}{l}\text { Los trabajadores (mano de obra, los empleados) } \\
\text { Los patronos (empresarios, gerentes) } \\
\text { Las organizaciones gubernamentales o privadas }\end{array}$ & $\begin{array}{l}\text { Constituye los agentes sociales articulados del } \\
\text { acto de la producción social organizado en } \\
\text { clases sociales (obreros y patronos). El Estado } \\
\text { expresa los intereses del sector dominante. }\end{array}$ \\
\hline $\begin{array}{l}\text { Proceso } \\
\text { Clave }\end{array}$ & $\begin{array}{l}\text { Transformación de los insumos a través de la } \\
\text { estructuración de unidades negociadoras, uso de } \\
\text { los mecanismos de presión en compensaciones } \\
\text { económicas, sociales y psicológicas que revierten } \\
\text { el entorno. Los insumos constituyen todo tipo de } \\
\text { demandas, aspiraciones, peticiones devenidas } \\
\text { internamente o del ambiente. }\end{array}$ & $\begin{array}{l}\text { Dialéctica implícita en el proceso de control } \\
\text { sobre la relación laboral expresado en términos } \\
\text { de regulación, desorden, pacto, alianzas. Interés } \\
\text { fundamental en el análisis histórico del } \\
\text { agregado social obrero, en su proceso } \\
\text { constructivo de su identidad de conciencia, la } \\
\text { valoración política del control sobre el espacio } \\
\text { de trabajo, la construcción de las alternativas de } \\
\text { cambio. }\end{array}$ \\
\hline El entorno & $\begin{array}{l}\text { Constituye el continente condicionador del } \\
\text { Sistemas de relaciones Industriales eso lo } \\
\text { conforma el subsistema ecológico, el subsistema } \\
\text { económico, el subsistema político, el legal, el } \\
\text { socio cultural. }\end{array}$ & $\begin{array}{l}\text { La formación socioeconómica, la formación } \\
\text { capitalista en sus distintas etapas y } \\
\text { modalidades. }\end{array}$ \\
\hline $\begin{array}{c}\text { Dinámica } \\
\text { del Sistema }\end{array}$ & Morfogénico, tensión, distención, acuerdos & $\begin{array}{l}\text { La dialéctica de regulación, desorden. El orden } \\
\text { socioeconómico, sus contrataciones históricas. } \\
\text { La conformación de la conciencia de clase } \\
\text { como criterio de control. }\end{array}$ \\
\hline
\end{tabular}

Fuente: Elaboración propia basada en Delgado (2004)

Estos dos modelos nos hacen reflexionar sobre sí ¿Son pertinentes los modelos contemporáneos de gestión de Recursos Humanos en sistemas no capitalistas? La teoría nos indica que la base de los sistemas socialistas/comunistas está basada en una dialéctica que refuerza la desaparición de las clases, lo cual no ayuda a formar sistemas de recompensas, ni desarrollo. La tendencia es el control a través de la conciencia de clase. Esto dista la visón capitalista que piensa en recompensas, meritocracia, desarrollo, libertad empresarial. El papel del Estado es distinto en cada sistema. Por ende podría decirse que los modelos contemporáneos de la GRRHH no son pertinentes en sistemas comunistas y podrían adaptarse en sistemas socialistas que tengan apertura de negocio, pero requerirán de muchas modificaciones para insertarse en la teoría socialista.

Además, el pensamiento social de las Relaciones Laborales, en ambos modelos es muy distinto. En el modelo cibernético se observa mayor apertura, orden, valoración del trabajador. 
Una visión donde la empresa tiene valor, hay organización del trabajo mecanismos de presión en compensaciones económicas, sociales y psicológicas que revierten el entorno. Es un modelo que permite pensar en elementos ecológicos, humanos; que miran dentro y fuera de la empresa entendiendo que hay una relación irrompible entre la institución y su medio ambiente.

En el caso del Modelo de cambio Social Histórico Dialectico, se puede observar que el pensamiento social de las Relaciones Laborales el Estado es el sector dominante y con mayores intereses, donde no se promueve el desarrollo de la empresa privada con la lucha de clases. Aspectos importantes la regulación, el desorden, la creación de alianzas, la valoración política. Marx (2014) pensaba que la dialéctica ayudaría a la libertad del hombre y a que el trabajo fuese apreciado de otra manera por su capacidad liberadora. Sin embargo, eso no se ha logrado.

En el caso Venezuela, con la aparición del chavismo (pensamiento político ligado al Marxismo) que buscó la construcción del Socialismo del Siglo XXI, el Estado ha tomado control de las empresas, lo cual ha incidido en la productividad de las mismas y su desaparición. Muchas se encuentran paradas o produciendo al mínimo de su capacidad. En este modelo la meritocracia no tiene cabida, lo que se emplea es el oportunismo. El tema de la inamovilidad laboral, ha hecho que sea muchas veces imposible lograr acuerdos para aumentar la productividad en las empresas. El sindicalismo se ha desvirtuado, así como los sistemas de compensaciones y protección ante las políticas del Estado. El Gobierno actual, a través del discurso, ha hecho creer que el trabajador es el elemento más importante, por la dialéctica marxista. Sin embargo, cuando se estudian los sistemas de compensaciones y protección, no sucede así.

Ante el panorama actual, es casi imposible hablar de evaluaciones de desempeño, esto hace que el esfuerzo en adiestramiento si se da, no puede ser medido para saber su efectividad. Los planes de carrera pueden ser escasos o no existir. Muchas veces se considera que el desarrollo es un gasto y no es necesario, ante el criterio de igualdad. El Estado se ha vuelto el gran empleador; las medidas adoptadas por el gobierno no ayudan al desarrollo de las empresas públicas. Fenómenos actuales como el bachaqueo, producto de las medidas regulatorias del gobierno, hace que las personas no quieran trabajar en la empresa y que la educación y desarrollo sea subestimado.

Otro elemento a tomar en cuenta es la calidad. Dentro de las empresas socialistas existentes, tanto la calidad como la productividad existen. Dado los sistemas de compensaciones existentes, los sueldos no alcanzan para pagar personas preparadas que puedan incidir en la calidad de los procesos y la producción.

En este sentido, podría decirse que en el caso Venezuela, las medidas tomadas por el Gobierno Nacional hace que sea imposible que los modelos contemporáneos de Gestión de Recursos Humanos sean viables y una opción para incorporar a las empresas. El atraso en el cual se vive hace que tengamos que pensar en modelos de GRRHH obsoletos, que se adapten a la realidad de las empresas privadas venezolanas y de las organizaciones públicas, manejadas por el mismo Estado.

\section{A modo de reflexión}


La Gestión de Recursos Humanos depende en gran medida del modelo económico imperante en el país. Las visiones sobre las cuales estos modelos montan sus paradigmas influyen profundamente en el que hacer de las organizaciones y en la concepción de sus valores, misión, visión y concepción de la institución en sí. En este sentido, es necesario que los profesionales de las Relaciones Laborales construyan prácticas efectivas que los ayuden a gestionar a la gente dentro de las organizaciones, de manera que estas logren avanzar y crecer bajo sistema en el que operan -capitalista o socialista-.

El caso de Venezuela representa reto importante para las profesionales del área, ya que requieren innovar, vencer obstáculos e impulsar a la gente al desarrollo de sus capacidades, para hacer que las organizaciones puedan avanzar bajo el modelo del Socialismo del Siglo XXI; entendiendo que en este país aún subsisten trasnacionales, empresas grandes y un parque industrial empequeñecido que debe seguir produciendo dentro del modelo imperante y que requiere de la gente para su desarrollo.

\section{Bibliografía}

Definición ABC. (2015a). Definición de Marx. Disponible en: http://www.definicionabc.com/historia/marx.php

Definición ABC. (2015b). Definición de Socialismo. Disponible en: http://www.definicionabc.com/politica/socialismo.php

Delgado, Yamile. (2014). Gestión de Recursos Humanos, Referencia Sector Manufacturero carabobeño. Tesis doctoral para optar al Título de Doctor en Ciencias Sociales, mención Estudios del Trabajo. Universidad de Carabobo.

Marx, Karl. (2014). El Capital Tomo I. E-FOCUS.

Vilchez, Carlos. (2011). Si el capitalismo anda mal, el socialismo no está mejor. Disponible en: http://www.carlosvilcheznavamuel.com/si-el-capitalismo-anda-mal-el-socialismo-noesta-mejor/

Weber, Max. (1978). El origen del capitalismo moderno. Disponible en: Historia económica general. México: F.C.E.

Werther, William, Davis Keith. (1991). Administración de recursos humanos. Mc Graw Hill. México 